(C)2013 American Physical Society. Access to this work was provided by the University of Maryland, Baltimore County (UMBC) ScholarWorks@UMBC digital repository on the Maryland Shared Open Access (MD-SOAR) platform.

Please provide feedback

Please support the ScholarWorks@UMBC repository by emailing scholarworks-group@umbc.edu and telling us

what having access to this work means to you and why it's important to you. Thank you. 


\title{
gs \\ Ultralow-power nonlinear optics using tapered optical fibers in metastable xenon
}

\author{
T. B. Pittman, D. E. Jones, and J. D. Franson \\ Department of Physics, University of Maryland Baltimore County, Baltimore, Maryland 21250, USA
}

(Received 26 August 2013; published 6 November 2013)

\begin{abstract}
We demonstrate nanowatt-level saturated absorption using a subwavelength diameter tapered optical fiber (TOF) suspended in a gas of metastable xenon atoms. This ultralow-power nonlinearity is enabled by a small optical mode area propagating over a relatively long distance through the Xe gas. The use of inert noble gases in these kinds of TOF experiments may offer practical advantages over the use of reactive alkali-metal vapors, such as rubidium.

DOI: 10.1103/PhysRevA.88.053804

PACS number(s): 42.65.-k, 42.81.Qb, 42.62.Fi, 42.50.Gy
\end{abstract}

Subwavelength diameter tapered optical fibers (TOFs) and small hollow-core photonic-band-gap fibers (PBGFs) enable low-loss propagation of evanescent and air-guided modes with very small mode areas over very long distances [1,2]. The interaction of these highly confined fields with atomic vapors can allow the realization of optical nonlinearities at remarkably low power levels [3]. For example, TOFs surrounded by rubidium vapor and PBGFs filled with rubidium vapor have recently been used to demonstrate saturated absorption, two-photon absorption, and a variety of other nonlinear effects at nanowatt- and even "few-photon-" power levels [4-10].

Unfortunately, the tendency of $\mathrm{Rb}$ to accumulate on silica surfaces [11] severely limits the performance of these devices. In the case of TOFs, Rb accumulation causes a drastic loss of transmission [12], whereas, in PBGFs, it can limit the penetration depth into the hollow core to several cm's [10]. The observation of these difficulties suggests the use of inert noble gases, rather than reactive $\mathrm{Rb}$ vapor, to improve these systems. Here we specifically investigate the use of xenon in TOF experiments. We observe saturated absorption at nanowatt-power levels, which indicates the suitability of this system for further ultralow-power nonlinear optics applications.

An overview of one particular set of Xe energy levels for these applications is shown in Fig. 1. A weak electric discharge is used to excite $\mathrm{Xe}$ to the $6 s[3 / 2]_{2}$ metastable state, which has a long intrinsic lifetime of $\sim 43 \mathrm{~s}$ [13]. This metastable state serves as an effective "ground state" for an optical ladder transition at 823 and $853 \mathrm{~nm}$ that can then be used for the various two-photon nonlinearities. The transition rates of these lines are $3 \times 10^{7}$ and $2 \times 10^{6} \mathrm{~s}^{-1}$, respectively, which are comparable to those of the well known $5 S_{1 / 2} \rightarrow 5 P_{3 / 2} \rightarrow 5 D_{5 / 2}$ ladder transition at 780 and $776 \mathrm{~nm}$ in $\mathrm{Rb}$ [14].

The purpose of this initial work was to perform saturation spectroscopy of the 823-nm transition using a TOF surrounded by a relatively low-density gas of metastable Xe atoms. The ability to saturate this transition at ultralow-power levels is an indicator of the overall strength of the atom-field interaction in this system.

Figure 2 shows a measured transmission spectrum of the 823-nm line obtained by passing a narrow-band tunable diode laser through a conventional $\mathrm{Xe}$ discharge tube used as a reference cell. The six primary transmission dips are due to contributions from the nine stable isotopes of natural Xe [15]. The largest dip (labeled dip 4) contains contributions from all seven even isotopes, whereas, the other dips are due to hyperfine splittings of ${ }^{129} \mathrm{Xe}$ and ${ }^{131} \mathrm{Xe}$ [16]. The reference cell data are used to calibrate the spectra observed in our main TOF system.

An overview of the main experimental apparatus is shown in Fig. 3. TOFs with a minimum waist diameter of $250 \mathrm{~nm}$ (and a sub-500-nm diameter over a length of $\sim 1 \mathrm{~cm}$ ) were produced using the flame-brush technique [17]. For the 823-nm wavelength of interest, this TOF size guides an evanescent field with a diameter on the order of $1 \mu \mathrm{m}$ over a length of approximately $1 \mathrm{~cm}$ [2]. This provides an effective "lengthto-area" ratio $\left(L_{\mathrm{eff}} / A\right)$ for nonlinear optical effects that is 3 to 4 orders of magnitude larger than comparable free-space focusing to a $1-\mu \mathrm{m}$ spot size.

The TOFs were mounted on a thin 3-in.-long cylindricalshaped Teflon frame using low-outgassing UV-curable epoxy. The TOF mounting jig was then inserted into a 12-in.-long glass discharge tube system composed of standard 1.33in. mini-ConFlat flanges and fittings. An electrical power feedthrough (nickel rod) was used as the discharge tube cathode, and a two-hole fiber feedthrough [18] was used for the input and output fiber leads. Xe pressures of 1 Torr were used for these experiments. A dc power supply (roughly $-1 \mathrm{kV}$ ) in series with an $8.2-\mathrm{k} \Omega$ ballast resistor was used to maintain the discharge at currents of $\sim 1 \mathrm{~mA}$.

As shown in Fig. 3, a free-space probe beam (derived from the primary laser) passed perpendicularly through the discharge tube to monitor the metastable Xe density during the TOF experiments. The probe and TOF output signals were simultaneously recorded as the laser was swept through the Xe resonances.

Figure 4 shows an example result obtained with an estimated power of $51 \mathrm{nW}$ passing through the TOF. The interaction of the TOF evanescent field with the surrounding metastable Xe atoms is apparent by the resonance absorption dips at the same locations as the free-space probe (the measurements were not sensitive enough to observe small shifts or transit-time line broadening effects). We use the largest dip (dip 4) to quantify our measurements of TOF transmission ( $86 \%$ in this example). When the run was repeated with larger powers in the TOF, the dip- 4 transmission was seen to increase towards unity, thereby demonstrating saturation of the system. 


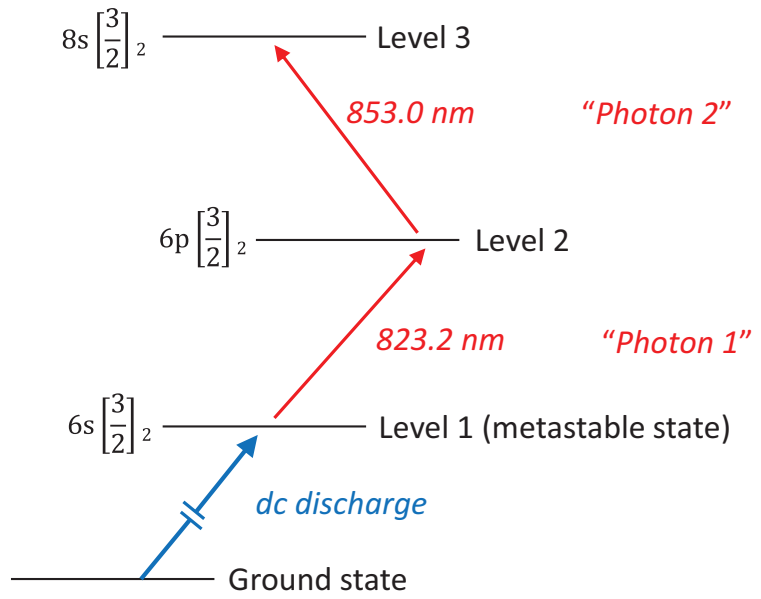

FIG. 1. (Color online) Diagram of the relevant xenon energy levels. A dc (or rf) discharge is used to excite the Xe atoms to a long-lived metastable state (denoted level 1). A two-photon ladder transition at 823 and $853 \mathrm{~nm}$ can then be used for the experiments of interest.

Figure 5 summarizes this saturation effect. The plot shows the measured TOF dip-4 transmission values for seven different powers in the range of $10-1000 \mathrm{nW}$. The power in the TOF waist region was estimated by assuming the intrinsic TOF loss (74\% for this particular fiber) was uniformly distributed over the entire tapered region and modifying the measured output power accordingly. The error bars are due to small uncertainties in maintaining a constant metastable Xe density (as measured by the free-space probe) and noise in the detection system.

In order to estimate the saturation power of our system, we fit the data in Fig. 5 to a standard Beer's law transmission model $T=e^{-\alpha_{n l} L}$ using a simple nonlinear absorption coefficient defined as $\alpha_{n l}=\alpha_{0} /\left(1+P / P_{\text {sat }}\right)$ with a linear absorption coefficient $\alpha_{0}[19,20]$. The blue curve shows this nonlinear transmission model with a best-fit saturation power of $P_{\text {sat }}=$ $(126 \pm 44) \mathrm{nW}$. For comparison, the dashed red line shows ordinary linear transmission with the same value of $\alpha_{0}$. This clear observation of nonlinear behavior (saturated absorption) at nanowatt-power levels in Fig. 5 is the main result of this paper. The estimated value of $P_{\text {sat }}=(126 \pm 44) \mathrm{nW}$ is

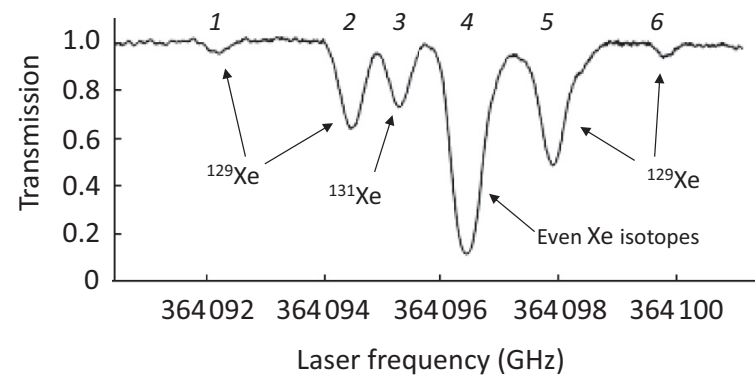

FIG. 2. Transmission spectrum of metastable Xe at $823 \mathrm{~nm}$ obtained using an auxiliary reference discharge cell. The dips labeled 1-6 are due to the various stable isotopes of natural Xe. The largest dip (labeled 4) is due to contributions from all of the even isotopes.

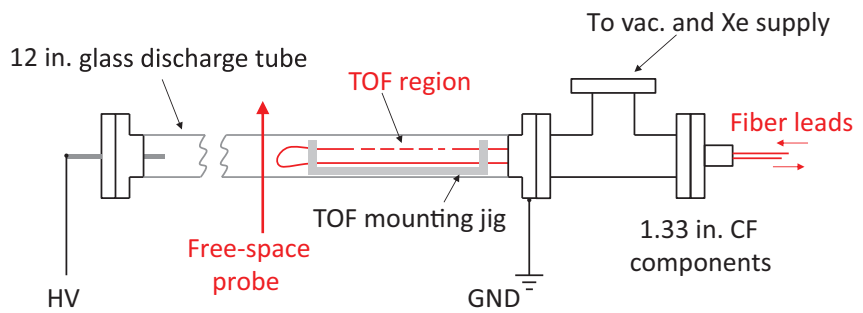

FIG. 3. (Color online) Design of the de discharge tube containing a TOF. A free-space probe beam passing perpendicularly through the discharge tube is used to monitor the density of metastable Xe atoms during the experiments.

comparable to a saturation power of $72 \mathrm{nW}$ that we recently measured with a similar TOF system in Rb vapor [12].

The fitted transmission value of $84 \%$ in Fig. 5 (at powers far below saturation) gives an overall TOF system optical depth (OD) of 0.17 . This corresponds to a relatively low metastable state atomic density in the system. We were able to obtain higher metastable state densities in our system by increasing the dc discharge power but typically observed a large and irreversible loss of TOF transmission as a result. This was presumably due to sputtering of nickel and other contaminants from the electrical feedthrough onto the TOF surface [21]. Even at the relatively weak discharge conditions used in our experiments, we typically observed a temporary transmission reduction of roughly $15 \%$ while the discharge was on, most likely due to contaminants passing through the evanescent field. We anticipate that these problems could be minimized by using a better "right-angle" dc discharge tube design or a standard rf discharge system [22,23].

Achieving higher ODs comparable to those typically used in related $\mathrm{Rb}$ experiments [4-10] will be a key next step for this system. The production of high Xe metastable state densities can be limited by Penning ionization and other de-excitation mechanisms [15]. The highest reported values $\left(\sim 10^{13} \mathrm{~cm}^{-3}\right)$ are typically observed in small-volume high-pressure pulsed

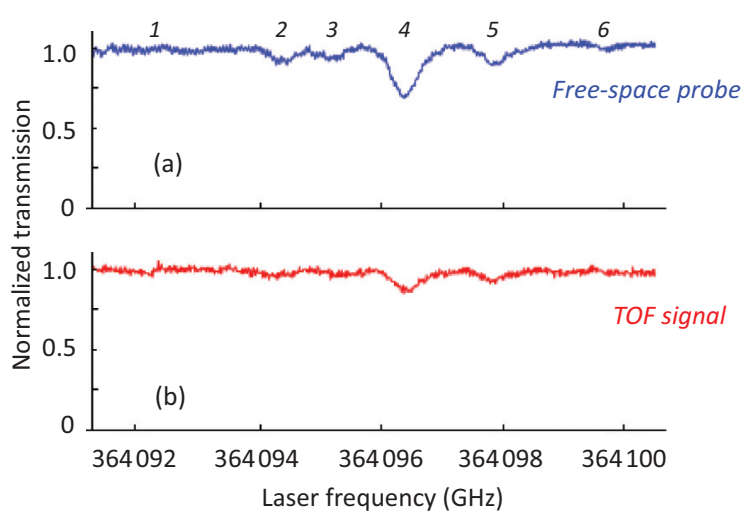

FIG. 4. (Color online) Transmission spectrum of metastable Xe at $823 \mathrm{~nm}$ using the apparatus shown in Fig. 3. Plot (a) shows the spectrum of the free-space probe beam passing perpendicularly through the discharge tube, whereas, plot (b) shows the spectrum simultaneously obtained by passing a $51-n W$ signal through the TOF. The absorption dips are labeled 1-6 for comparison with the reference cell data of Fig. 2. The traces in (a) and (b) are normalized to their overall transmission values far from the Xe resonances. 


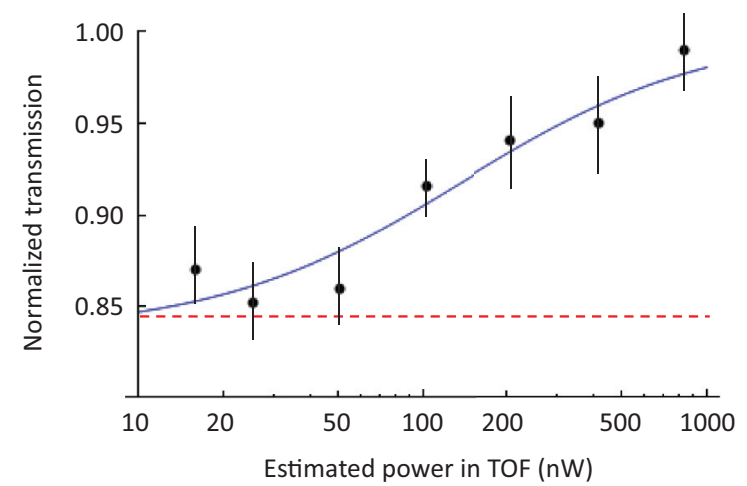

FIG. 5. (Color online) Measured TOF transmission as a function of increasing power passing through the TOF. The data points are best fit to a simple nonlinear transmission model (blue line) with an ultralow saturation power of $(126 \pm 44) \mathrm{nW}$. For comparison, the dashed red line shows what would be expected from ordinary linear transmission.

discharge systems [24], whereas, values of $\sim 10^{10} \mathrm{~cm}^{-3}$ are more typical in the steady-state low-intensity discharge systems [16] considered here. The ability to generate higher densities without damaging the TOFs will facilitate the realization of practical ultralow-power nonlinear optics devices based on this system. Producing large metastable state Xe densities confined in small-core PBGFs may present additional challenges but is also worthwhile due to the extremely long interaction lengths that may be possible.

To summarize, we have observed nanowatt-level saturated absorption using a TOF suspended in a relatively low-density gas of metastable Xe atoms. This initial study suggests the use of metastable $\mathrm{Xe}$ gas as a promising alternative to hot $\mathrm{Rb}$ vapor for ultralow-power nonlinear optics experiments using TOFs. The two-photon ladder transition of Fig. 1 has a number of desirable properties, and the nanowatt-level saturation of the lower transition observed here indicates the suitability of the system for various "two-beam" applications $[6,10]$. Achieving higher ODs (through higher Xe metastable state densities) will be a key next step in realizing these important applications using this system.

This work was supported by DARPA DSO under Grant No. W31P4Q-12-1-0015.
[1] R. F. Creegan, B. J. Mangan, J. C. Knight, T. A. Birks, P. S. J. Russell, P. J. Roberts, and D. C. Allan, Science 285, 1537 (1999).

[2] L. Tong, J. Lou, and E. Mazur, Opt. Express 12, 1025 (2004).

[3] R. Boyd, Nonlinear Optics (Academic, New York, 1992).

[4] S. Ghosh, A. R. Bhagwat, C. K. Renshaw, S. Goh, A. L. Gaeta, and B. J. Kirby, Phys. Rev. Lett. 97, 023603 (2006).

[5] S. M. Spillane, G. S. Pati, K. Salit, M. Hall, P. Kumar, R. G. Beausoleil, and M. S. Shahriar, Phys. Rev. Lett. 100, 233602 (2008).

[6] S. M. Hendrickson, M. M. Lai, T. B. Pittman, and J. D. Franson, Phys. Rev. Lett. 105, 173602 (2010).

[7] K. Saha, V. Venkataraman, P. Londero, and A. L. Gaeta, Phys. Rev. A 83, 033833 (2011).

[8] V. Venkataraman, K. Saha, P. Londero, and A. L. Gaeta, Phys. Rev. Lett. 107, 193902 (2011).

[9] K. Salit, M. Salit, S. Krishnamurthy, Y. Wang, P. Kumar, and M. S. Shariar, Opt. Express 19, 22874 (2011).

[10] V. Venkataraman, K. Saha, and A. L. Gaeta, Nat. Photonics 7, 138 (2012).

[11] J. Ma, A. Kishinevski, Y.-Y. Jau, C. Reuter, and W. Happer, Phys. Rev. A 79, 042905 (2009).
[12] M. M. Lai, J. D. Franson, and T. B. Pittman, Appl. Opt. 52, 2595 (2013).

[13] M. Walhout, A. Witte, and S. L. Rolston, Phys. Rev. Lett. 72, 2843 (1994).

[14] A. Kramida et al., NIST Atomic Spectra Database (National Institute of Standards and Technology, Gaithersburg, MD, 2013) [www.nist.gov/pml/].

[15] M. Walhout, H. J. L. Megens, A. Witte, and S. L. Rolston, Phys. Rev. A 48, R879 (1993).

[16] T. Xia, S. W. Morgan, Y.-Y. Jau, and W. Happer, Phys. Rev. A 81, 033419 (2010).

[17] T. A. Birk and Y. W. Li, J. Lightwave Technol. 10, 432 (1992).

[18] E. R. L. Abraham and E. A. Cornell, Appl. Opt. 37, 1762 (1998).

[19] W. Demtroder, Laser Spectroscopy (Springer-Verlag, Berlin, 1982), Chap. 2.

[20] A. Yariv, Quantum Electronics (Wiley, New York, 1989).

[21] M. Fujiwara, K. Toubara, and S. Takeuchi, Opt. Express 19, 8596 (2011).

[22] C. Y. Chen et al., Rev. Sci. Instrum. 72, 271 (2001).

[23] C. I. Sukenik and H. C. Busch, Rev. Sci. Instrum. 73, 493 (2002).

[24] H. S. Uhm, P. Y. Oh, and E. H. Choi, Appl. Phys. Lett. 93, 211501 (2008). 